\title{
OPTIMISING THE START-UP OF A 10Hz BOOSTER POWER SUPPLY
}

\author{
S A Griffith, P Dickenson \& R J Smith \\ CLRC, Daresbury Laboratory, Daresbury, Warrington, WA4 4AD, UK
}

\section{Abstract}

The Synchrotron Radiation Source (SRS) at Daresbury Laboratory has a $600 \mathrm{MeV}$ Booster, fed by an energy storage power supply configuration (White Circuit). The design consists of two power supplies : an AC source to supply the losses to the parallel resonant circuits and a DC supply to fully bias the AC sinewave. In a recent modernisation of the control electronics, different methods of achieving reliable synchronisation of the AC source on switch-on have been investigated. The circuitry must deal with the high $\mathrm{Q}$ value of the resonant circuits and variation in resonant frequency with both ambient temperature and the applied DC current. This paper presents the different designs explored to improve the efficiency of start-up and discusses the problems encountered. The chosen design and test results are detailed with recommendations for future investigations.

\section{INTRODUCTION}

The majority of Booster AC power converter breakdowns are overcurrent trips, which occur during turn on, resulting in and eventual fuse and possible

Table 1: Range of frequencies providing successful turn-on. (Y- success, X- Failure)

\begin{tabular}{|c|c|c|}
\hline Internal & Turned ON & Changed Over \\
\hline Frequency & Internal Oscillator & To Resonant \\
\hline 9.810 & $\mathrm{X}$ & $\mathrm{X}$ \\
\hline 9.822 & $\mathrm{X}$ & $\mathrm{X}$ \\
\hline 9.834 & $\mathrm{X}$ & $\mathrm{X}$ \\
\hline 9.846 & $\mathrm{X}$ & $\mathrm{X}$ \\
\hline 9.860 & $\mathrm{X}$ & $\mathrm{X}$ \\
9.866 & $\mathrm{Y}$ & $\mathrm{Y}$ \\
9.871 & $\mathrm{Y}$ & $\mathrm{Y}$ \\
9.879 & $\mathrm{Y}$ & $\mathrm{Y}$ \\
9.885 & $\mathrm{Y}$ & $\mathrm{Y}$ \\
9.890 & $\mathrm{Y}$ & $\mathrm{Y}$ \\
9.896 & $\mathrm{Y}$ & $\mathrm{X}$ \\
\hline 9.910 & $\mathrm{Y}$ & $\mathrm{X}$ \\
\hline 9.924 & $\mathrm{Y}$ & $\mathrm{X}$ \\
\hline 9.945 & $\mathrm{Y}$ & $\mathrm{X}$ \\
\hline 9.970 & $\mathrm{Y}$ & $\mathrm{X}$ \\
\hline 9.984 & $\mathrm{Y}$ & $\mathrm{X}$ \\
\hline 9.998 & $\mathrm{X}$ & $\mathrm{X}$ \\
\hline
\end{tabular}

thyristor faults. The power converter technology is a conventional thyristor inverter generating the $10 \mathrm{~Hz}$ sinewave, with output current stability controlled by a three-phase thyristor rectifier supplying DC to the inverter.

To start the system the power converter initially forces the 'White circuit' to oscillate at a frequency slightly higher than its resonant frequency. This is achieved with an internal oscillator and the system operating in open loop. Once a significant feedback signal is obtained from the 'White circuit', frequency and amplitude closed loop control can be applied.

The high $\mathrm{Q}$ value of the circuit makes the choice of internal frequency critical to a successful turn-on of the supply. The results in Table 1 show that the internal frequency has to be within a band of $0.118 \mathrm{~Hz}$ to guarantee start up in open loop. A narrow window of $0.024 \mathrm{~Hz}$ is then available for the transition to frequency feedback. If a frequency outside this range is selected the power converter fails on overcurrent.

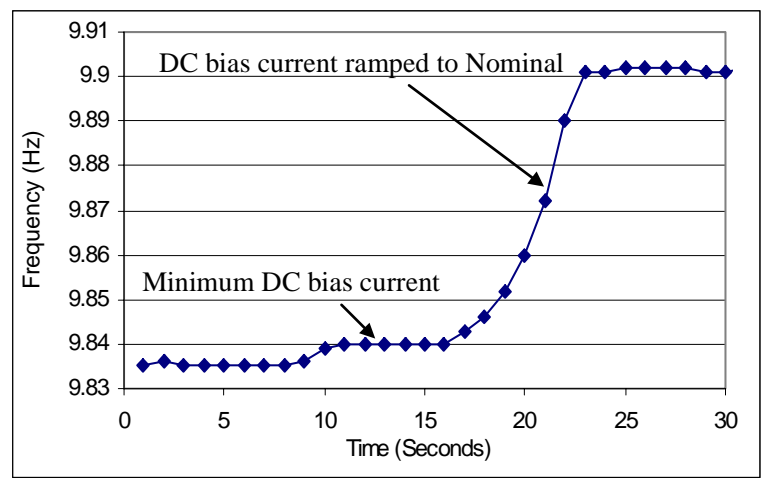

Figure 1: Change of resonant frequency with DC bias.

\section{INFLUENCES ON RESONANCE}

Ideally if the resonant frequency remained stable an internal oscillator with a stability of $9.88 \mathrm{~Hz}+/-0.012$ would guarantee turn-on. In reality the frequency is dependent on ambient temperature of both oscillator and 'White circuit' and the DC bias current. The results in Fig. 1 show that the frequency varies by $0.065 \mathrm{~Hz}$ when the DC current is applied. This is outside the allowable operating range in Table 1 , and there is no internal frequency that can be set to ensure 100\% success. 


\section{DESIGN OPTIONS}

The simplest solution is to set a fixed internal frequency and program the start up sequence so that the AC supply is turned on before the DC. This is not ideal and the superior method is to use an intelligent controller that can ensure turn-on irrespective of the external factors. A programmable integrated circuit (PIC) 16C715 - 20 was chosen to explore the different design options, as it is flexible and programs can be easily modified. There was five solutions considered:

- Internal oscillator - optimised frequency;

- Start pulse - optimised frequency;

- Continuous pulse with direct sychronisation;

- Start pulse burst with reduced delay;

- Extended pulse burst firing.

\subsection{Internal oscillator - optimised frequency}

The majority of turn-on failures occur during the transition from open to closed loop control. This method ensures a successful transition by measuring the resonant frequency while operating in open loop, allowing the optimum internal frequency to be set before the transition. When running in open loop the internal and feedback signals are locked in frequency, but their phase displacement shown in Fig. 2 is directly related to the resonant frequency. This displacement can easily be measured and the new start-up frequency calculated and set automatically.

- Disadvantages - the system will still fail if the internal frequency is initially set wrong and there is a significant delay between evaluating optimum frequency and eventual turn on.

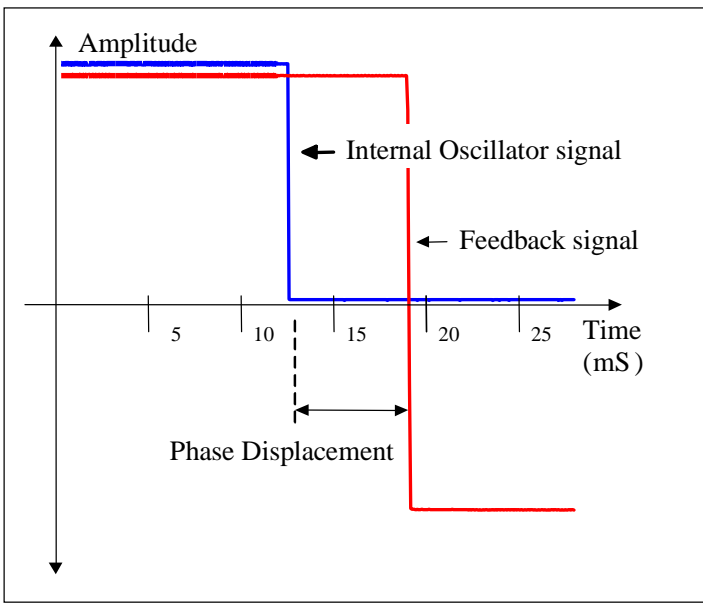

Figure 2: Phase displacement is directly related to resonant frequency

\subsection{Start pulse - optimised frequency}

This attempts to measure the resonant frequency without using the internal oscillator, preventing initial start up problems. The principle is to fire the inverter once to generate a minimum of 25 feedback cycles as shown in Fig.3, enabling the resonant frequency to be measured and the optimum internal frequency set. The accuracy of the result is dependent on the time displacement between firing each leg of the inverter and on the pulse number the measurement is taken.

- Disadvantage - testing showed the frequency measurement was difficult to set up and had poor repeatability. A compensation factor had to be added to calculate the required frequency due to the low test AC current.

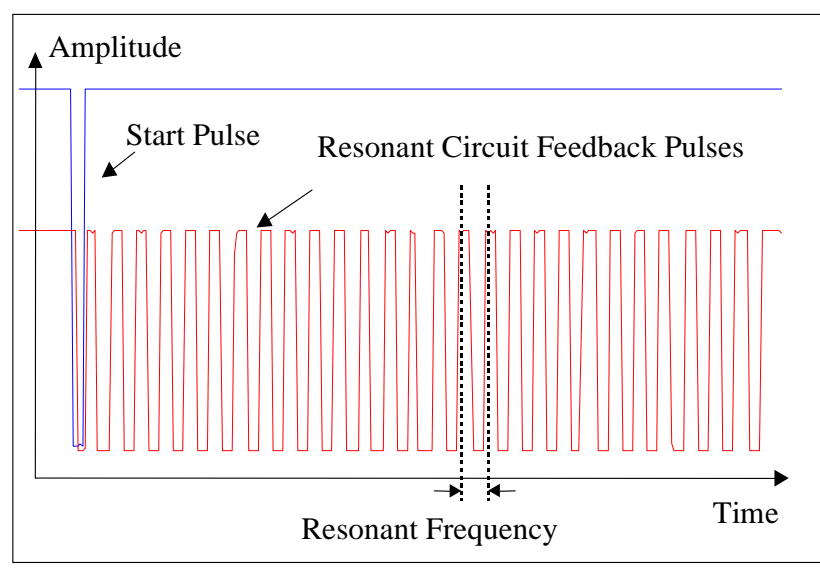

Figure 3: Feedback cycles generated from a start pulse.

\section{$3.3 \quad$ Continuous pulse with direct synchronisation}

This method again fires the inverter once generating feedback signals, but immediately provides continuous firing pulses synchronised to the feedback signal. This achieves fast stabilisation of the output current and optimises turn-on time. During testing the circuit could, on average operate for only seven cycles before the power supply tripped on overcurrent as shown in Fig.4. A low percentage of turn on successes were recorded.

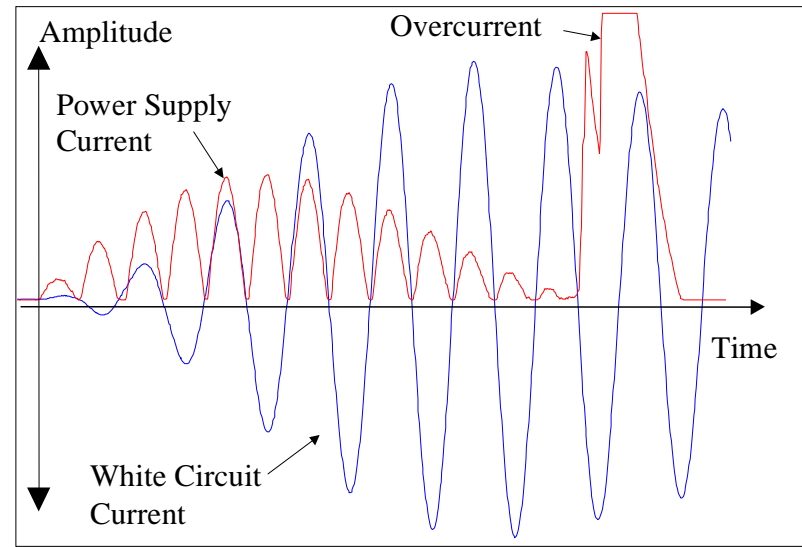

Figure 4: Direct Synchronisation with overcurrent trip. 
The preliminary evidence suggests that this was due to the control loop characteristics and with further investigations this might of been be resolved, but this was not explored due to time constraints.

\subsection{Start Pulse Burst with Reduced Delay}

This solution is based on increasing the 'White Circuit' current slowly before applying continuous feedback. This is achieved by firing the inverter once at intervals, with a gradual reduction in delay. The number of singular pulses applied and their intervals can be programmed for optimisation. The examples shown in Fig. $5 \& 6$, are for nine and seven single inverter pulses applied with a linear decrease in the space between pulses, with each pulse synchronised with the feedback signal. The reliability of this design varied considerably depending on the settings; even after optimisation the success rate could not be improved beyond $80 \%$.

- Disadvantage - significant time taken to reach stable output current and turn-on could not be guaranteed.

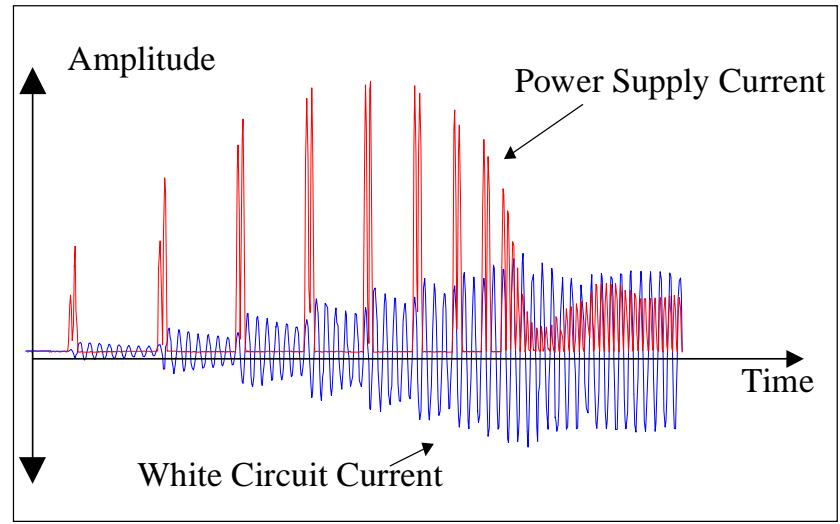

Figure 5: Nine single start pulse bursts with reducing delay.

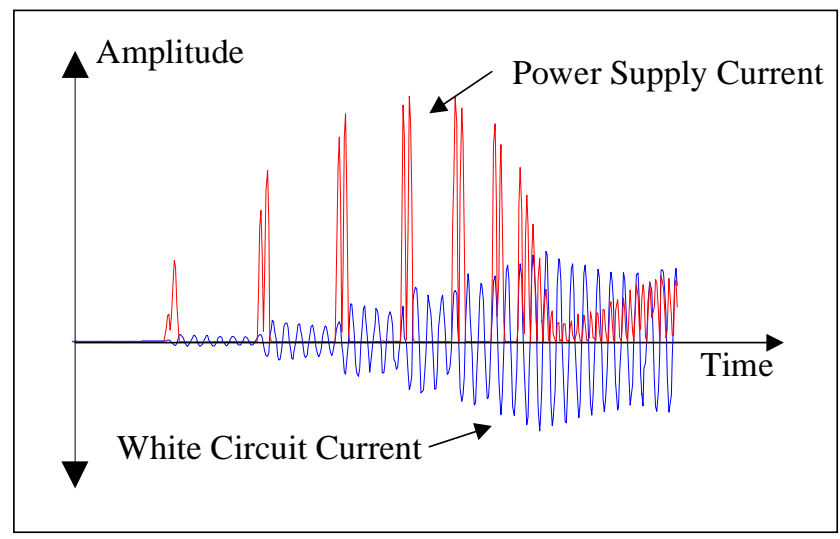

Figure 6: Seven single start pulse bursts with reducing delay.

\subsection{Extended Pulse Burst Firing}

This was the method finally implemented. The principle is to control the number of pulses applied during each burst, (not singular as in previous method) before closed loop control initiated. After some optimisation the sequence demonstrated in Fig. 7 provided the best results and shows a three pulse bursts, with a linear reduction in delay. This allowed a smooth transition to closed loop control that was repeatable and guaranteed a successful turn-on. As there is a gradual increase of 'White circuit' current towards nominal before the transition, problems with the control loop characteristics are compensated. There are no disadvantages with this method and a number of variations on the start-up sequence could be employed to achieve the same result.

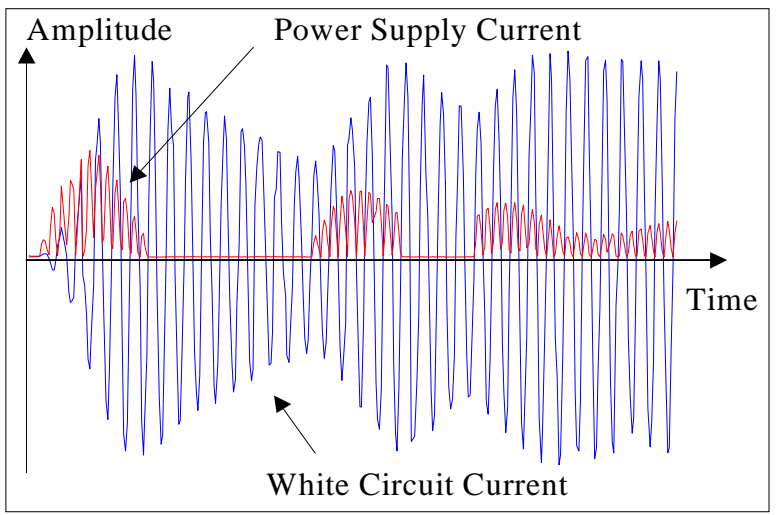

Figure 7: Extended Pulse burst firing with reducing delay.

\section{FUTURE DEVELOPMENTS}

In addition to the Pulse burst firing sequence program there will be a diagnostic program, which includes a single pulse application and an internal oscillator option. These can be used to identify potential power electronic faults, if turn on problems were encountered.

A liquid crystal display is to be installed to provide information for diagnostics and general measurements such as the resonant frequency.

Investigations into improving the performance of the power converter control loops to develop the direct synchronisation method are also planned.

\section{SUMMARY}

This design has been operational for 9 months and has proved to be effective and reliable. There has been a significant improvement in operational efficiency with only one failure during this period due to a faulty PIC, which failed during testing.

The power converter can now be turned on irrespective of ambient temperature, DC bias current and the high $\mathrm{Q}$ value of the resonant circuits. A number of viable solutions to turning on the power converter of a 'White circuit' have been explored. All the solutions proposed could be developed, but realistically the continuous pulse with direct synchronisation has the most potential. 